\title{
An Optical Sensor for Tracking Hand Articulations
}

\author{
Wang L, Meydan T, and Williams P \\ Wolfson Centre for Magnetics, School of Engineering, Cardiff University, Cardiff, Wales, UK \\ Email: Meydan@cardiff.ac.uk
}

\begin{abstract}
Recognizing and tracking articulations of the human hand is key to the development of areas such as robotics, virtual reality systems and physical rehabilitation. Based on the principle of crossed-polarization detection, a novel optical sensor, based on a hinge configuration, is proposed to monitor finger articulation. Using 3D printing technology, we fabricated a lightweight, compact sensor suited to attaching on fingers. The weighted average method was applied to the sensor's output data to determine angular positions corresponding to finger joint articulations. The experimental results showed excellent consistency with theoretical predictions. The sensor features good accuracy $( \pm 0.5 \%$ of full-scale $)$ and repeatability, improved sensitivity, and an improved measuring range of $180^{\circ}$. The performance of the sensor is a promising development for monitoring finger articulation. Future work will focus on integrating multiple sensors as part of an instrumented glove to evaluate the true potential for monitoring hand articulation.
\end{abstract}

Keywords—optical sensor; polarization; hand motion tracking; $3 D$ printing; sensing techniques; rehabilitation.

\section{INTRODUCTION}

Since the 1970's, hand motion tracking has gained considerable attention in both academia and industry $[1,2]$. Various sensing technologies including resistance flex sensors [3], optical fibre techniques [4], magnetic sensing elements [5], and Inertial Measurement Units (IMU) [6], have been investigated for capturing hand movements. Many of these methods either lack accuracy or require complex and timeconsuming calibration procedures. Sensors may also possess a limited angular range and/or suffer from significant drift errors. There is a clear need to address these problems by developing new improved sensing technologies.

We have previously reported the use of a polarization technique for monitoring hand articulation [8]. Although some rotational sensors or encoders using polarization techniques have been presented elsewhere [8, 9], they have focused on military or commercial aircraft applications and not human motion tracking. In our previous work, we presented an optical sensor based on the principle of crossed-polarization detection. The measuring range of $90^{\circ}$, however, is not sufficient to monitor the full range of articulation in metacarpophalangeal (MCP) and proximal interphalangeal (PIP) joints. Secondly, the sensitivity is not constant but varies as a function of angle.

To tackle the above problems, we describe an improved optical sensor, based on a hinge design, with an expanded measuring range of $180^{\circ}$. The sensor's design, characterization method and performance results will be discussed in the following sections.

This research is jointly sponsored by China Scholarship Council (CSC) and Cardiff University.

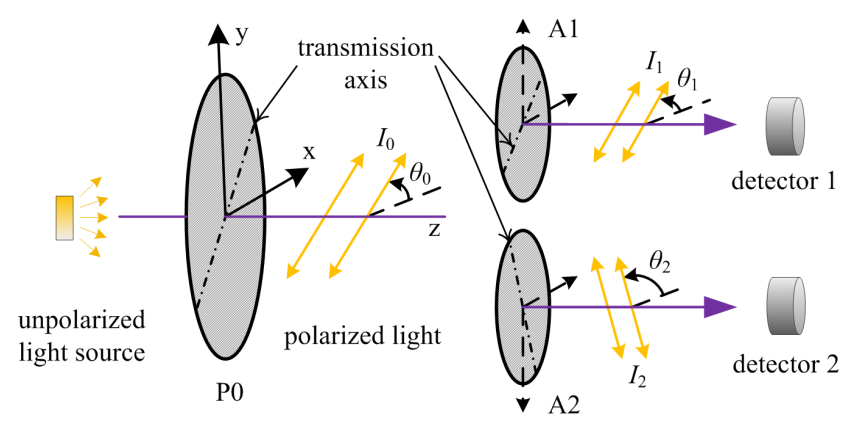

Fig. 1. The schematic diagram of the polarized optical sensor.

\section{SENSOR DESIGN}

\section{A. Principle of operation}

As shown in Fig. 1, the optical sensor consisted of a single light source, a linear polarizer P0, two analyzers A1 and A2, and two photodetectors. Passing through $\mathrm{P} 0$, the incident light was polarized in the direction of the transmission axis at an angle $\theta_{0}$ to the $\mathrm{x}$-axis and then split into two separate channels. Each channel has its own analyzer and photodetector providing simultaneous outputs. The two analyzers, A1 and A2, are oriented with their transmission axes at $45^{\circ}$ degrees to each other. This ensures that at least one sensor will be operating in the region with maximum sensitivity and linearity.

According to Malus' Law, the light intensity $I_{1}$ and $I_{2}$ should obey the following theoretical relationships:

$$
\begin{aligned}
& I_{1}=I_{0} \times \cos ^{2}\left(\theta_{1}-\theta_{0}\right) \\
& I_{2}=I_{0} \times \cos ^{2}\left(\theta_{2}-\theta_{0}\right)
\end{aligned}
$$

where $I_{0}$ is the initial polarized light intensity and $\theta_{1}$ and $\theta_{2}$ are the transmission angles, relative to the $\mathrm{x}$-axis, for analyzers $\mathrm{A} 1$ and $\mathrm{A} 2$ respectively.

\section{B. Fabrication Details}

Inspired by the articulated structure of finger joints, we developed a hinge type configuration for our optical sensor. The sensor was fabricated using an EnvisionTEC's Perfactory Mini Multi Lens 3D Printer using RCP30 resin. Fig. 2 (a) shows a photograph of the integrated optical sensor with a diameter of $4.2 \mathrm{~mm}$ and a width of $10 \mathrm{~mm}$. The sensor was constructed with a range of dimensions, as shown in Fig. 2 (b). 


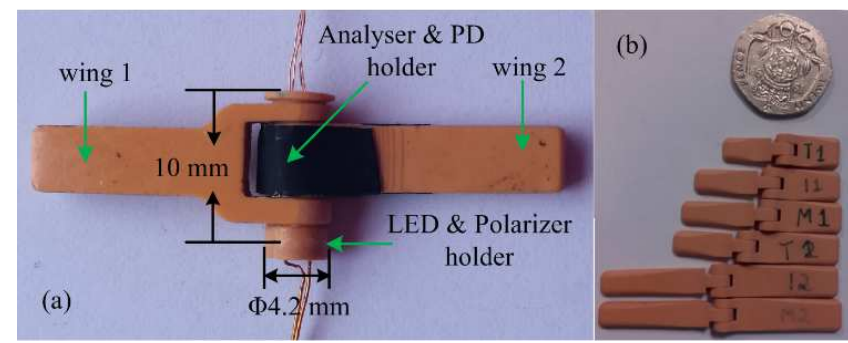

Fig. 2. Image of the complete sensor. (a) The integrated optical sensor. (b) A range of component sizes.

The sensor's polarizer and analyzers were made from commercial linear polarizing film. Two high sensitive PIN photodiodes (TEMD6200FX01) were employed to detect changes in light intensity. The LED source and the polarizer P0 were located inside a separate holder and located in wing 1 . The two analyzers and photodiodes (PD) were housed inside another holder located inside wing 2 . When the wings rotate, the angle between the polarizer and two analyzers also changes, leading to a change in light intensity. The final light intensities were linearly converted to an electrical current by the photodiodes and then to voltages by conditioning circuitry.

\section{MEASUREMENTS}

An automated experimental setup was employed to reduce operator errors, see block diagram in Fig. 3. A $360^{\circ}$ continuous motorized rotation stage NR360S (Thorlabs Inc.) [10] and a micro-stepping motor controller BSC201, was employed to adjust the rotation angles. Wing 2 of the optical sensor was fixed while wing 1 rotated under the guidance of the motorized stage. Overall system control was achieved using LabVIEW which involved implementing ActiveX interfacing technology for device control and preforming data acquisition using a National Instrument card (NI USB-6211).

The conditioning circuits converted the PD electrical current $i$, proportional to the illumination intensity $(I)$, to voltages $(V)$. Referring to (1) and (2), the voltages can be obtained by the following equations.

$$
\begin{aligned}
V_{1}=k_{1} \times i_{1}+b_{1}=m_{1} \times I_{1}+b_{1} & =m_{1} \times I_{0} \times \cos ^{2}\left(\theta_{1}-\theta_{0}\right)+b_{1} \\
& =a_{1} \times \cos ^{2}\left(\delta_{1}\right)+b_{1} \\
V_{2}=k_{2} \times i_{2}+b_{2}=m_{2} \times I_{2}+b_{2} & =m_{2} \times I_{0} \times \cos ^{2}\left(\theta_{2}-\theta_{0}\right)+b_{2} \\
& =a_{2} \times \cos ^{2}\left(\delta_{2}\right)+b_{2}
\end{aligned}
$$

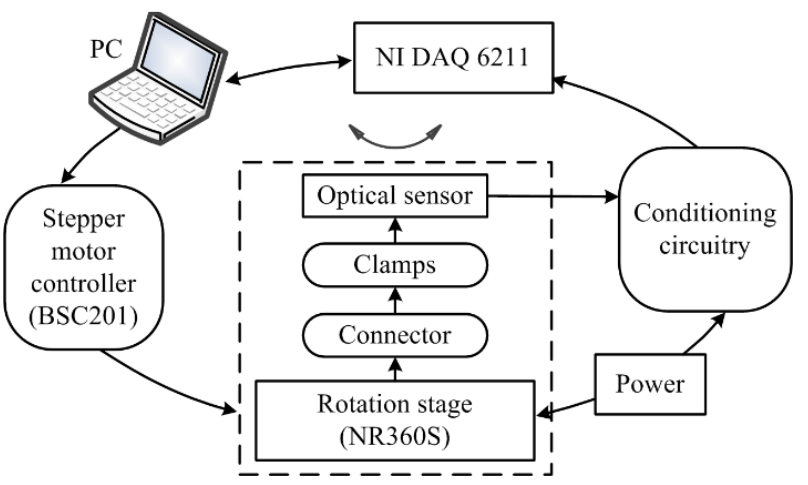

Fig. 3. The block diagram of the automated experimental set-up. where the subscripts ' 1 ' and ' 2 ' represent the parameters for Channel 1 and Channel 2, respectively. $\delta_{1}$ and $\delta_{2}$ are the rotation angles relative to the polarization direction of $\mathrm{P} 0$. The parameters $k_{i}, m_{i}$ and $a_{i}(i=1,2)$ are the constants of proportionality, and $b_{i}$ are constant voltages due to less than perfect blocking of light when the polarizers are set orthogonally.

In this paper, we define $0^{\circ}$ to be the position when the sensor wings are in line with each other.

The measurements were carried out at room temperature with the motor rotating at $10 \%$ and data sampling at $100 \mathrm{~Hz}$. The optical sensor was rotated from $-100^{\circ}$ to $90^{\circ}$, and then back to $-100^{\circ}$ in intervals of $5^{\circ}$. Each reading was composed of 500 samples. This process was repeated 5 times with an interval of 3 minutes between each cycle.

\section{RESUlTS AND DISCUSSIONS}

\section{A. Sensitivity and Hysteresis}

Averaging the data over five cycles, leads to a voltage-toangle relationship for both channels as plotted in Fig. 4. The theoretical values obtained from (3) and (4), were calculated and plotted as well.

The measured output voltages of both channels were consistent with the theoretical values. The maximum deviation from the theoretical voltages was $15 \%$ and occurred at the minimum outputs (approximately $60 \mathrm{mV}$ ). This is unsurprising since the signal is small and the sensitivity is a minimum. Additionally, both channels exhibited an identical response in both clockwise and anticlockwise directions with a hysteresis error less than $1.2 \%$. The measurement span was limited to $90^{\circ}$ for each channel, and the sensitivity was smallest at positions corresponding to maximum and minimum output readings.

\section{B. Angle Conversion}

To expand the measuring range and improve the sensor's sensitivity, we combined the information from two channels to obtain the angular position.

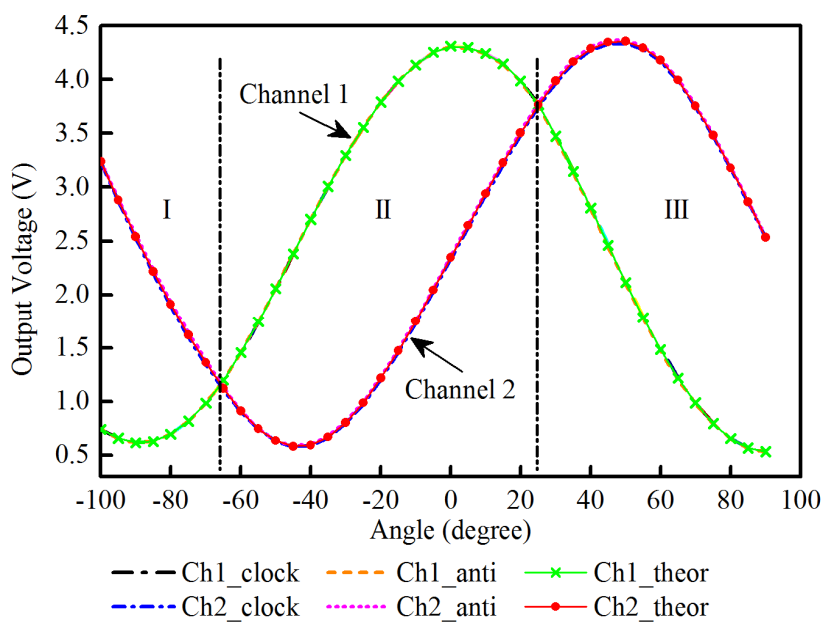

Fig. 4. The voltage-to-angle relationships of the optical sensor. Ch1_clock, Ch1_anti, and Ch1_theor represented the output readings from Channel 1 in clockwise and anticlockwise directions, and the theoretical values. Similarly, the Ch2_clock, Ch2_anti, and Ch2_theor were from Channel 2. 
According to the amplitudes of both channels, the output voltages can be divided into three regions, I, II, and III (see Fig. 4). In region I and region III, Channel 1 output is smaller in amplitude compared to Channel 2. The opposite is true in region II. By comparing the amplitudes of each channel, we can select the channel operating in the linear region for that specific angle. By differentiating between the two channels in this way, we can effectively have a linear output spanning $180^{\circ}$ combined with high sensitivity.

In order to avoid a high dependency on one channel, the weighted average method was used to calculate the angular position. For each channel, the rotation angles $\delta_{1}$ and $\delta_{2}$ can be obtained by (3) and (4). Therefore, the final angle $\delta$ will be:

$$
\delta=w_{1} \times \delta_{1}+w_{2} \times \delta_{2}
$$

where $w_{1}$ and $w_{2}$ were the allocated different weights for Channel 1 and Channel 2, respectively.

There are various approaches to determine the weights. Considering the varying sensitivities in both channels, we allocated variable weights for $\delta_{1}$ and $\delta_{2}$ which could be calculated by (6) and (7). At each bending position (angle of articulation), the weights were determined by the closeness of the readings to the voltage at $45^{\circ}$.

$$
\begin{gathered}
w_{1}=\left(1-\frac{\left|V_{1}-V_{1}\left(\delta_{1}=45\right)\right|}{\left|V_{1}-V_{1}\left(\delta_{1}=45\right)\right|+\left|V_{2}-V_{2}\left(\delta_{2}=45\right)\right|}\right)^{3 / 2} \\
w_{2}=1-w_{1}
\end{gathered}
$$

The converted sensor angle measured at each test position is shown in Fig. 5. The error bars for the output angle, too small to be seen in Fig.5, are less than $0.1^{\circ}$ and were determined from a standard uncertainty multiplied by a coverage factor of 3 , which provides a confidence level of $99.7 \%$. The data in Fig. 5 shows that the sensor has good full-scale accuracy $( \pm 0.5 \%)$ and excellent repeatability over the full $180^{\circ}$ measurement range.

\section{CONCLUSION}

We have demonstrated by doubling the number of sensing elements in our optical sensor we can extend the measuring range to $180^{\circ}$ compared to that achieved with our previous sensor. This enables the sensor to monitor the entire range of movement experienced by PIP, MCP, and other articulated joints in the human body. The accuracy and the sensitivity has also been improved by adopting the weighted average approach.

The 3D printing technique used to fabricate the sensor achieved a diameter of $4.2 \mathrm{~mm}$ and a width of $10 \mathrm{~mm}$. This is sufficiently compact to be suitable for use on the hands of most adults. The optical sensor therefore has great potential for use as sensing elements in devices for hand motion tracking. In the future, we intend to focus on integrating this sensor into an instrumented glove system for real-time monitoring of hand motions.

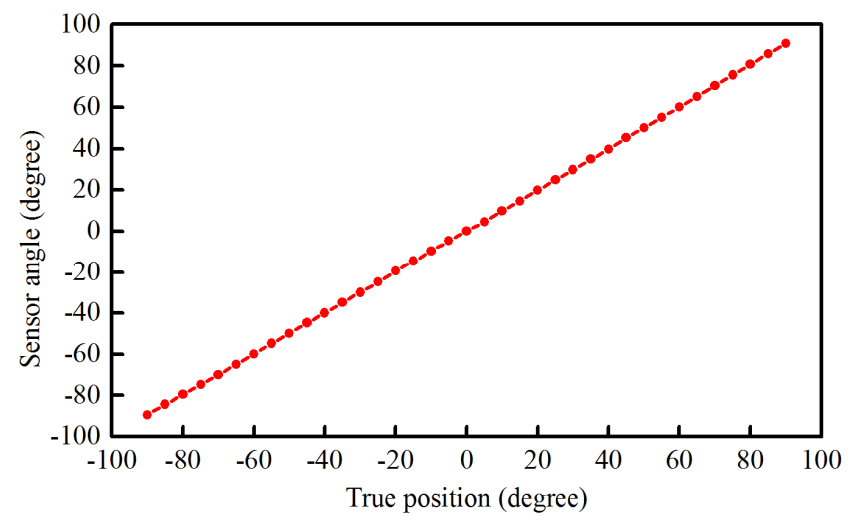

Fig. 5. The converted sensor angles versus the true position.

\section{REFERENCES}

[1] L. Dipietro, A. M. Sabatini, and P. Dario, "A survey of glove-based systems and their applications," IEEE trans. Syst., Man, Cybern. C, Appl. Rev., vol. 38, no. 4, pp. 461-482, July 2008.

[2] D. J. Sturman and D. Zeltzer, "A survey of glove-based input," IEEE Comput. Graph. Appl., vol. 14, no. 1, pp. 30-39, January 1994.

[3] M. Amjadi, A. Pichitpajongkit, S. Lee, S. Ryu, and I. Park, "Highly stretchable and sensitive strain sensor based on silver nanowire-elastomer nanocomposite," ACS Nano., vol. 8, no. 5, pp. 5154-5163, April 2014.

[4] A. F. S. Silva, A. F. Gonc salves, P. M. Mendes, and J. H. Correia. "FBG sensing glove for monitoring hand posture," IEEE Sens. J., vol. 11, no. 10, pp. 2442-2448, October 2011.

[5] H. G. Kortier, J. Antonsson, H. M. Schepers, F. Gustafsson, and P. H. Veltink, "Hand Pose Estimation by Fusion of Inertial and Magnetic Sensing Aided by a Permanent Magnet," IEEE Trans. Neural Syst. Rehabil. Eng., vol. 23, pp. 796-806, 2015.

[6] H. G. Kortier, V. I. Sluiter, D. Roetenberg, and P. H. Veltink, "Assessment of hand kinematics using inertial and magnetic sensors," J. Neuroeng. Rehabil., vol. 11, pp. 70, 2014.

[7] L. Wang, T. Meydan, P. Williams, and T. Kutrowski, "A proposed optical-based sensor for assessment of hand movement," Sens. 2015 IEEE, Busan, pp. 1-4, November 2015.

[8] G. J. Wijntjes and C. T. Markos, "Non-contact optical polarization angle encoder," U.S. Patent 2005/0 002 032, Jan. 6, 2005.

[9] N. Albion, R. F. Asmar, R. W. Huggins, G. E. Miller, C. R. Porter, "Optical angle sensor using polarization techniques," U.S. Patent 5424 535, Jun. 13, 1995.

[10] Thorlabs, Inc., [online], Available: http://www.thorlabs.co.uk/newgrouppage9.cfm?objectgroup_id=1064\& $\mathrm{pn}=\mathrm{NR} 360 \mathrm{~S} / \mathrm{M} \# 2605$. 